Article published in: Hydrogen in Matter: A Collection from the Papers Presented at the 2nd International Symposium on Hydrogen in Matter; ISOHIM, AIP Conference Proceedings Volume 837, American Insitute of Physics (2006) 12 - 21

\title{
Hydrogen Retention In Plasma-Sprayed Tungsten
}

\author{
A.V. Golubeva ${ }^{1,2}$, V.A. Kurnaev ${ }^{1}$, M. Mayer ${ }^{2}$, J.Roth ${ }^{2}$ \\ 1 - Moscow Physics' and Engineering Institute, Moscow, Russia \\ 2- Max-Planck-Institut für Plasmaphysik, EURATOM Association, Boltzmannstr. 2, D-85748 Garching, \\ Germany
}

\begin{abstract}
Deuterium retention in plasma-sprayed tungsten (PSW) was investigated by means of the thermodesorption technique. The material was irradiated by deuterium ions with energies of $200 \mathrm{eV}$ and $3 \mathrm{keV}$ per deuteron, achieving fluences in the range $10^{22} \div 10^{24} \mathrm{D} / \mathrm{m}^{2}$. The observed retention in PSW is four times higher than in polycrystalline tungsten (PCW). An additional high-temperature peak was found in the thermodesorption spectra of PSW (at $1050 \mathrm{~K})$. The nature of the high-temperature peak and the mechanism of higher retention in PSW are discussed.
\end{abstract}

Keywords: hydrogen, retention, tungsten, plasma-sprayed tungsten

PACS: $52.40 \mathrm{Hf}$, 81.05.Bx, 81.05.Rm.

\section{INTRODUCTION}

Thermonuclear fusion is a possible energy source for the future. The international experiment ITER is intended to show the ability of getting energy from fusion in a reactor with magnetic confinement of the plasma. It is an intermediate step to a DEMO reactor, and at this step technologies, materials and methods will be tested and optimized.

Tritium accumulation in first wall materials is of interest and importance from both fuel balance and radiation safety points of view. Three materials are foreseen to be used for plasma-facing (PF) surfaces in ITER: Beryllium, graphite and tungsten [1]. The use of carbon might result in unacceptably high tritium inventories due to tritium co-deposition. To decrease the tritium inventory in PF materials it was proposed [2,3] to exchange all plasma-facing materials to tungsten. Even in the current design, the area covered with tungsten will be no less than $140 \mathrm{~m}^{2}$ [1]. One method for covering such a large area with tungsten is plasma spraying of tungsten on some feasible substrate.

Tungsten coated graphite tiles were used at different regions (divertor strike points, central column, auxiliary limiters, etc.) of the tokamak experiment ASDEX Upgrade in several experimental campaigns [4, 5]. Plasma spraying of tungsten turned 
out to be a suitable method of tungsten layer deposition for tokamak application [6]. The advantages of plasma spraying in comparison with other deposition methods are homogeneously distributed voids, which stop crack propagation under high heat loads.

Nowadays there exists a substantial database on hydrogen-tungsten interaction, but at the same time there are a lot of unsolved problems. The amount of hydrogen retained and peculiarities of retention strongly depend on the material structure, which in turn depends on the material production procedure [10]. Most experiments in this field were performed with polycrystalline (PCW) or monocrystalline (SCW) tungsten, while data for plasma-sprayed tungsten (PSW) are very scarce and far from being complete.

The deuterium inventory in plasma-sprayed tungsten coatings on graphite divertor tiles of ASDEX Upgrade was analyzed after 800 plasma discharges and 6 boronizations [7]. It was found that in some areas the tungsten was covered with a deposit consisting mainly of carbon, boron and hydrogen isotopes. The total deuterium inventory was found to be between $4 \cdot 10^{21}$ and $3 \cdot 10^{23} \mathrm{D} / \mathrm{m}^{2}$. The authors concluded that deuterium trapping in ASDEX Upgrade tiles is dominated by deuterium co-deposition with boron and carbon. In [8] the amount of retained deuterium was measured as a function of tile position. The highest inventory (up to $5 \cdot 10^{22} \mathrm{D} / \mathrm{m}^{2}$ ) was observed in the divertor region outside the separatrix due to the formation of a-C:H layers on the tungsten surface. Because the deuterium inventory in tokamak experiments is mostly determined by codeposition with light elements, it is difficult to derive any information about deuterium inventories in tungsten from these measurements.

Laboratory data on hydrogen interaction with PSW are very scarce $[9,10]$.

The investigation in [9] was performed for ASDEX Upgrade before the first tungsten divertor campaign in order to predict the deuterium capture in plasmasprayed tungsten. In this work trapping of deuterium implanted with an incident energy of $100 \mathrm{eV} / \mathrm{D}$ into polycrystalline and two different sorts of plasma-sprayed tungsten (produced by Plansee-AG and CEN-Cadarache) were investigated by means of re-emission and thermodesorption techniques. The authors compared the retention in these materials for a fluence of $10^{22} \mathrm{D} / \mathrm{m}^{2}$. For this case they found that retention in PCW is two times lower than in PSW produced by Plansee-AG and three times lower than in PSW by CEN-Cadarache. The difference in trapping was associated with different porosity of the samples, which was less than 1\% for PCW, 8-9\% for PSW by Plansee-AG and 15-20\% for PSW CEN-Cadarache. By analysing thermodesorption spectra, the authors concluded that annealing up to $600 \mathrm{~K}$ is enough to release all trapped deuterium.

Deuterium retention in five different tungsten materials including PSW was investigated in [10]. Samples were irradiated by $4.5 \mathrm{keV} \mathrm{D}_{3}{ }^{+}$ions at $300 \mathrm{~K}, 600 \mathrm{~K}$ and $900 \mathrm{~K}$ up to fluences of $\geq 10^{22} \mathrm{D} / \mathrm{m}^{2}$, and re-emission experiments were performed. The deuterium inventory was calculated from the difference between implanted and re-emitted fluencies. For samples irradiated at room temperature TDS measurements were carried out. For selected fluencies at room temperature D capture in SCW and PSW was found to be almost similar, while trapping in hot-rolled tungsten was three times higher. With increasing temperature the deuterium inventory dropped and at $\mathrm{T}=900 \mathrm{~K}$ it was 50 times lower than at the same fluence at room temperature. The 
ratio of deuterium retained in SCW, PSW and PCW rests 1:1:3 in the whole temperature interval.

So, the data on hydrogen retention in PSW are very limited and partly contradictive, and a detailed analyses of hydrogen retention on plasma-sprayed tungsten is necessary.

\section{EXPERIMENTAL}

\section{Experimental Set-Up}

The experimental investigation of deuterium retention in tungsten materials was carried out at the „High ion current device“ [11]. A duopigatron ion source provides ion beams in the energy range below $8 \mathrm{keV}$. The ion beam is mass separated with a magnetic sector field.

The tungsten samples were irradiated by deuterium at two energies: $\mathrm{D}_{3}{ }^{+}$with $E_{0}=600 \mathrm{eV}$ and $\mathrm{D}_{2}{ }^{+}$with $\mathrm{E}_{0}=6 \mathrm{keV}$, corresponding to $200 \mathrm{eV}$ per deuteron and $3 \mathrm{keV}$ per deuteron. To a first approximation one may neglect the molecular effect and can suppose that after molecular dissociation at the sample surface the energy divides between the atomic fragments of the molecular ion equally. In order to obtain higher ion fluxes in the case of low-energy irradiation $3.6 \mathrm{keV} \mathrm{D}_{3}{ }^{+}$ions were extracted from the ion source and decelerated to $600 \mathrm{eV}$ by a positive sample potential. A fraction of the incident ions can be neutralized at the entrance apertures, and this energetic neutrals are not decelerated by the sample potential. In previous works $[12,13]$ the fraction of high-energy neutrals in the decelerated beam was found to be 1.5 to $3 \%$, resulting in an admixture of a high energy component with $1.2 \mathrm{keV} / \mathrm{D}$. This led to a $10 \%$ uncertainty of the low-energy particles fluence retained [14]. Ion implantations were always performed at normal incidence. Without additional heating sample temperatures were about room temperature in the low-energy irradiation experiments, while the sample temperature was about $170^{\circ} \mathrm{C}$ in the case of $6 \mathrm{keV} \mathrm{D}{ }^{+}$implantation due to sample heating by the ion bombardment. The samples could be additionally heated by electron impact. The sample temperature was measured by infrared pyrometer. In both cases of high- and low-energy irradiation the flux at the sample surface was about $10^{20} \mathrm{D} / \mathrm{m}^{2} \mathrm{~s}$ at an implantation area of $0.16 \mathrm{~cm}^{2}$.

Deuterium retention in tungsten was investigated by means of the thermodesorption technique. After implantation the sample was heated with a linear heating ramp of $4.5 \mathrm{~K} / \mathrm{s}$. The signals of $\mathrm{D}_{2}, \mathrm{HD}$ and several hydrocarbon gases were registered by a quadrupole mass-analyzer, calibrated as described in [15] by deuterium capture and release in titanium. Depending on vacuum condition, the fraction of deuterium released as HD molecules is from 5 to $30 \%$. The fraction of deuterium atoms released as hydrocarbons is negligible because of good vacuum conditions. 


\section{Thermodesorption measurements}

For carrying out thermodesorption measurements of hydrogen retained in a material after ion implantation, it is necessary to extract the background signal (originating from the target holder, chamber walls etc.) from the measured intensity $\mathrm{I}(\mathrm{T})$. At the high-current ion source, the background originates from deuterium residing on constructive elements surrounding the target, from where it is released during after-irradiation sample annealing, because these elements are also slightly heated up to about $50^{\circ} \mathrm{C}$. This weak temperature rise is enough to release the residing $\mathrm{D}_{2}$ molecules and distort the deuterium release signal from the target. To separate the signal of deuterium thermally released from the sample from the spurious background signal the following four-steps TDS procedure was developed (fig. 1a).

After implantation the sample is extracted to atmosphere through a lock chamber and replaced by an identical, but not irradiated sample. This not irradiated sample is annealed by the normal thermodesorption ramp and the deuterium registered (curve 1) corresponds to molecules desorbed from surrounding surfaces. A second annealing after half-hour delay (curve 2) shows that all deuterium was released from these surfaces during the first annealing. Then the annealed sample is replaced by the original, irradiated sample and annealing of the irradiated sample is performed (curve 3 ). After half-hour delay the sample is annealed a second time (curve 4), and this second spectrum can be taken as background. The thermodesorption spectrum (TDS) is then the difference between the third and forth spectra (fig. 1b), multiplied with a calibration coefficient and plotted versus sample temperature.

Through this procedure the measurement of deuterium retained in tungsten was performed about 1 hour after the end of the implantation. During this time all mobile deuterium, which is dissolved in tungsten, is released from the sample, while deuterium trapped at lattice defects is almost not lost [10].

a

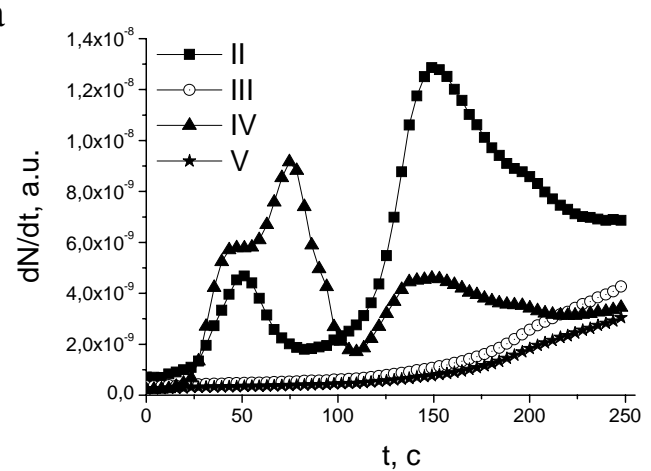

b

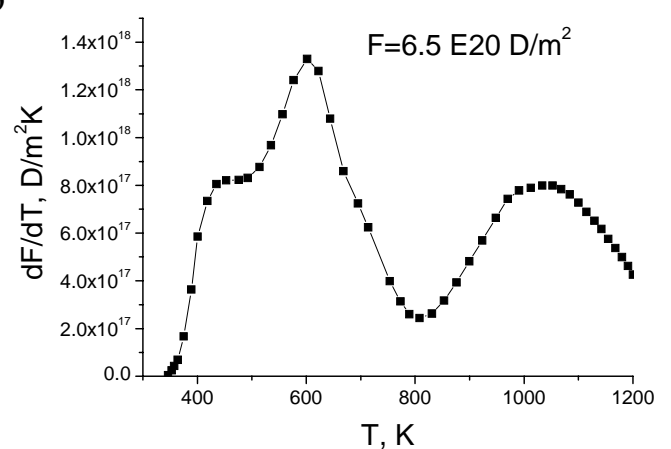

FIGURE 1. Evaluation of $\mathrm{D}_{2}$ signal in order to obtain the TDS of PSW after $3 \mathrm{E} 23 \mathrm{D} / \mathrm{m}^{2}$ irradiation by $200 \mathrm{eV} \mathrm{D}^{+}$at RT: a) Four-steps thermodesorption procedure: 1 - annealing with non-irradiated sample in order to desorb deuterium from surrounding surfaces, 2 - checking if all deuterium was desorbed, 3 linear annealing of irradiated sample, 4 - background registration, b) $\mathrm{D}_{2}$ signal as a function of sample temperature $\mathrm{T}$. 


\section{Materials under investigation}
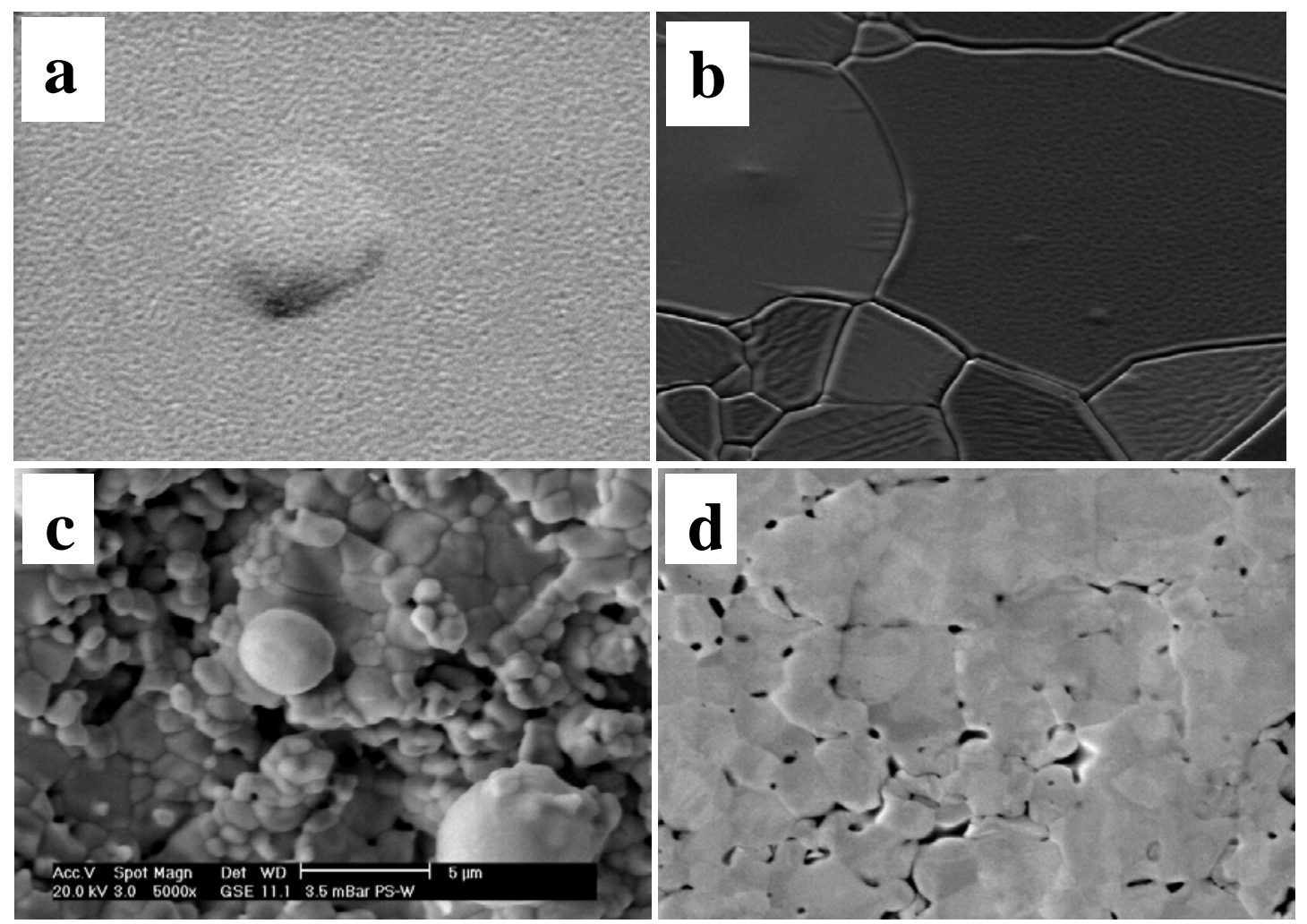

FIGURE 2. Materials under investigation. All SEM images have the same scale: a) monocrystalline tungsten surface, b) PCW surface, c) PSW surface, d) PSW cross-section

Deuterium retention was investigated in the following tungsten materials:

- plasma-sprayed tungsten (PSW) samples were cut from the front surface of an ASDEX Upgrade tile which was never exposed to plasma. The ASDEX Upgrade tiles consist of fine grain graphite with dimensions $160 \times 80 \times 30 \mathrm{~mm}$, of which the front surface is covered with $500 \mu \mathrm{m}$ tungsten deposited by high-density plasma spraying. To prevent carbon diffusion and tungsten carbide formation an intermediate layer of $10 \mu \mathrm{m}$ thick Re produced by physical vapor deposition is placed between carbon and tungsten.

- polycrystalline tungsten (PCW) samples were cut from $99.96 \%$ purity tungsten foils produced by Plansee-AG. They contain small amounts of $\mathrm{O}, \mathrm{C}, \mathrm{N}, \mathrm{H}, \mathrm{Fe}, \mathrm{Mo}, \mathrm{Ca}, \mathrm{Na}, \mathrm{Si}, \mathrm{P}$ impurities. After cutting the samples were polished, electro polished and pre-annealed in vacuum at $\mathrm{T}=1500$ $\mathrm{K}$ during three hours. This procedure reduced the number of intrinsic defects and led to grain sizes growth.

- monocrystalline tungsten (SCW) produced by State Institute of Rare Metals (Moscow) by double electron-beam zone melting. Samples were preliminary polished and annealed at the same conditions as PCW.

All samples had dimensions of about $10 \times 15 \mathrm{~mm}$ and thicknesses in the range $0.5 \div 1 \mathrm{~mm}$. SEM images of PSW surface in comparison with surfaces of PCW and 
SCW, and also PSW cut-off are shown in fig. 2. SCW has a perfect homogeneous structure corresponding to a single crystal. PCW consists of grains with sizes from $2 \div 50 \mu \mathrm{m}$. PSW has a very rough surface due to the plasma spray process. But as a result of baking at $1700 \mathrm{~K}$ after spraying cut-of of PSW (fig. $2 \mathrm{~d}$ ) shows a homogeneous structure without visible lamination. PSW sample has voids, which amount was estimated to be 8-9 \% of the volume [10]. The other investigated materials have no visible voids.

\section{RESULTS AND DISCUSSION}

\section{TDS Shapes}

Thermodesorption spectra of PCW and PSW irradiated with a fluence of $10^{24}$ $\mathrm{D} / \mathrm{m}^{2}$ of low-energy deuterium ions with $\mathrm{E}=200 \mathrm{eV} / \mathrm{D}$ are shown in fig. 3 .

In the thermodesorption spectrum (TDS) of tungsten (PCW) normally two peaks are present: At about $400 \mathrm{~K}$ and $650 \mathrm{~K}$. These peaks are related to traps with binding energies of about $0.5-0.9$ and 1.2-1.5 eV. Up to now there is no complete understanding of the nature of these traps. The first may be associated with some defects of the tungsten lattice (grain boundaries, dislocations, impurities, presence of bulk oxide [15]). Other authors suppose that this peak corresponds to capture at interstitial sites in the lattice [9]. The second peak is usually supposed to correspond to lattice defects created during ion bombardment (vacancies, bubbles), as it grows after high-energy ion pre-irradiation which creates displacement defects in tungsten.

Some authors also reported the existence of higher energy traps. Van Veen et al. [16] registered this peak at $800 \mathrm{~K}$ in the TDS of SCW irradiated by $200 \mathrm{eV}$ deuterons. Before deuterium implantation the sample was pre-implanted by $6 \mathrm{MeV}$ protons and $\varnothing 1 \mathrm{~nm}$ voids were created in the material. This peak was associated with deuterium release from small gas bubbles. In [17] the hydrogen binding energy in bubbles in SCW was found to be $2.1 \mathrm{eV}$, corresponding to an annealing temperature of $900 \mathrm{~K}$.

The TDS of PSW shows several indistinguishable peaks in temperature interval from 400 to $700 \mathrm{~K}$ (fig.2). In the present work an additional high-temperature peak around $1000 \mathrm{~K}$ is also observed in PSW, in contrast to PCW. As one can see from fig. $2 b$, the third high-temperature peak exists in the full range of investigated irradiation fluencies $10^{22}-2 \cdot 10^{24} \mathrm{D} / \mathrm{m}^{2}$.

TDS dynamics as a function of fluence (fig. 2b) shows that all three peaks grow proportional to each other with increasing fluence. During about 20 hours implantation (necessary to reach a fluence of $10^{24} \mathrm{D} / \mathrm{m}^{2}$ ) in tungsten even at room temperature deuterium may diffuse far beyond the implantation zone [18]. Consequently, increasing fluence we increase with $\mathrm{D}$ deeper tungsten layers. A proportional one to other increase of all three TDS peaks with increasing fluence let us suppose that the corresponding trap sites are constantly distributed over the PSW volume and are connected with some intrinsic features of PSW, which are results of the way of material manufacture, and not appeared as an ion induced effect. This let us to suppose 
that low-energy irradiation does not create additional trap sites in PSW. This is confirmed by identity of the thermodesorption spectra 1 and 3 in fig. 3b. These spectra were obtained for the same fluence of $200 \mathrm{eV}$ deuterons, but the TDS 1 was obtained before pre-implantation by $10^{24} \mathrm{D} / \mathrm{m}^{+}$of $\mathrm{D}^{+} 200 \mathrm{eV}$ while the TDS 3 - after. One can see that high fluence implantation of $200 \mathrm{eV}$ deuterons doesn't change trapping sites distribution. Deuterons of this energy cannot displace the atom of tungsten lattice and create a vacancy defect. And possible implantation doesn't led to additional gas filled voids creating as there already present sufficient amount of voids in PSW.

The TDS spectra of PSW show significantly broader peaks than TDS spectra of PCW. The reason may be the very rough surface of PSW.

So, the main possible reason of TDS peculiarity for PSW is its structure including very rough surface, spikes, voids (see fig. $2 \mathrm{~d}$ ). The latter can be the reason of the hightemperature TDS peak. In case of deuterium release from gas-filled voids in the sample, this process includes more than two steps: Thermodesorption of deuterium molecules from void surfaces with gas pressure increment (which increases with temperature rise), dissociation of deuterium molecules, atomic deuterium pushing into the $\mathrm{W}$ bulk, diffusion to outer surface, recombination on open surfaces with final deuterium desorption as molecules.
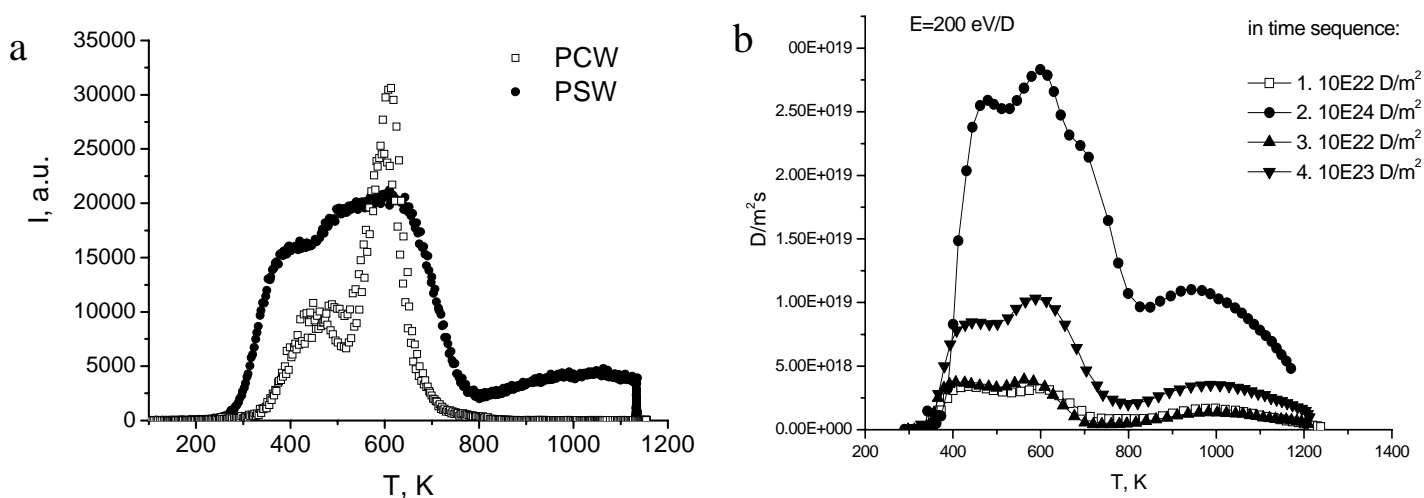

FIGURE 3. a) Thermodesorption spectra of PCW and PSW irradiated by $10^{24} \mathrm{D} / \mathrm{m}^{2}$ with an incident energy of $200 \mathrm{eV} / \mathrm{D}$, heating rate $0.5 \mathrm{~K} / \mathrm{s}$. b) thermodesorption spectra of one PSW sample implanted with fluences of $10^{22}, 10^{23}, 10^{24} \mathrm{D} / \mathrm{m}^{2}, \mathrm{E}_{0}=200 \mathrm{eV} / \mathrm{D}$, heating rate $5 \mathrm{~K} / \mathrm{s}$. Curve 3 was obtained for a sample, pre-implanted with $10^{24} \mathrm{D} / \mathrm{m}^{2}$

The high-temperature peak in the TDS of PSW is important for fusion reactor applications, because complete removal of tritium retained in the material needs heating to temperatures of at least $1250 \mathrm{~K}$, compared with polycrystalline tungsten where an annealing temperature of $800 \mathrm{~K}$ is sufficient. These results are in contradiction to [9]. The reason might be the more accurate background extraction procedure applied in the present work.

\section{Fluence Dependence of Deuterium Retention}

The fluence dependence of deuterium accumulation in PCW and PSW for ions with an initial energy of $200 \mathrm{eV} / \mathrm{D}$, as obtained by TDS measurements, is shown in fig. 4. In our experiments we didn't find saturation of deuterium trapping even for polycrystalline tungsten up to a fluence of $10^{24} \mathrm{D} / \mathrm{m}^{2}$. 
In the whole interval of fluences $10^{22} \div 10^{24} \mathrm{D} / \mathrm{m}^{2}$ deuterium retention in PSW is 4 times higher than in PCW. For the higher incident energy of $3 \mathrm{keV} / \mathrm{D}$ the difference in deuterium retention in PSW and PCW is the same. This difference is a result of material production leading to a much higher amount of lattice distortions in case of PSW. The higher retention in PSW is important for fusion applications, because this means four times higher tritium inventory.

In fig. $4 \mathrm{~b}$ the fluence dependence of deuterium accumulation in PSW for two incident energies $200 \mathrm{eV} / \mathrm{D}$ and $3 \mathrm{keV} / \mathrm{D}$ are shown. At low fluences deuterium capture is about three times higher for $3 \mathrm{keV} / \mathrm{D}$ energy, because of higher ranges and lower reflection coefficient at higher energies. According to SRIM simulations, the maximum range of deuterium implanted in tungsten is $100 \mathrm{~nm}$ for $\mathrm{E}_{0}=200 \mathrm{eV} / \mathrm{D}$ and $1000 \mathrm{~nm}$ at is at $\mathrm{E}_{0}=3 \mathrm{keV} / \mathrm{D}$, and deuterium reflection coefficients are equal to 0.65 and 0.45 for these two initial energies.

With increasing fluence the retention for $3 \mathrm{keV}$ incident energy grows more slowly than for $200 \mathrm{eV} / \mathrm{D}$ and at fluencies larger than $>10^{24}$ the amount of deuterium retained at $\mathrm{E}_{0}=200 \mathrm{eV}$ becomes equal to the amount retained at $3 \mathrm{keV}$ deuteron irradiation. With further increase of incident fluence we expect a higher retention for lower irradiation energy.

The higher trapping at lower implantation energies is most probably a temperature effect, because the $3 \mathrm{keV}$ ion beam heats the sample to temperatures of about $450 \mathrm{~K}$, while the sample temperature is close to room temperature at $200 \mathrm{eV}$ implantation. Higher temperatures result in a higher mobility of deuterium atoms in tungsten and in increased release from traps with low binding energy. The influence of sample temperature on hydrogen retention in PSW is illustrated in fig. 5, were thermodesorption spectra and total amounts of deuterium retained are given for several samples implanted with the same fluence but at different temperatures.
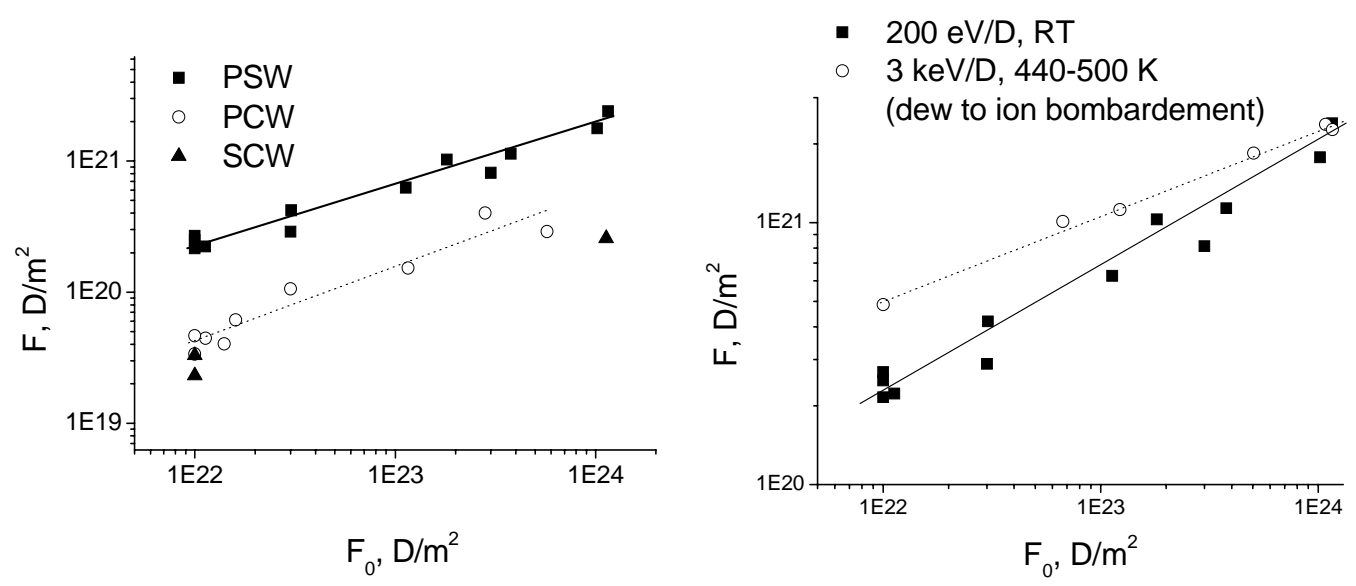

FIGURE 4. Fluence dependence of deuterium retention in PSW: a) - retention in PCW, SCW and PSW. $\left.E_{0}=200 \mathrm{eV} / \mathrm{D}, \mathrm{b}\right)$ - deuterium retention in PSW for two initial energies: $E_{0}=200 \mathrm{eV} / \mathrm{D}$ and $E_{0}=3$ $\mathrm{keV} / \mathrm{D}$. Lines are given to guide the eyes. 

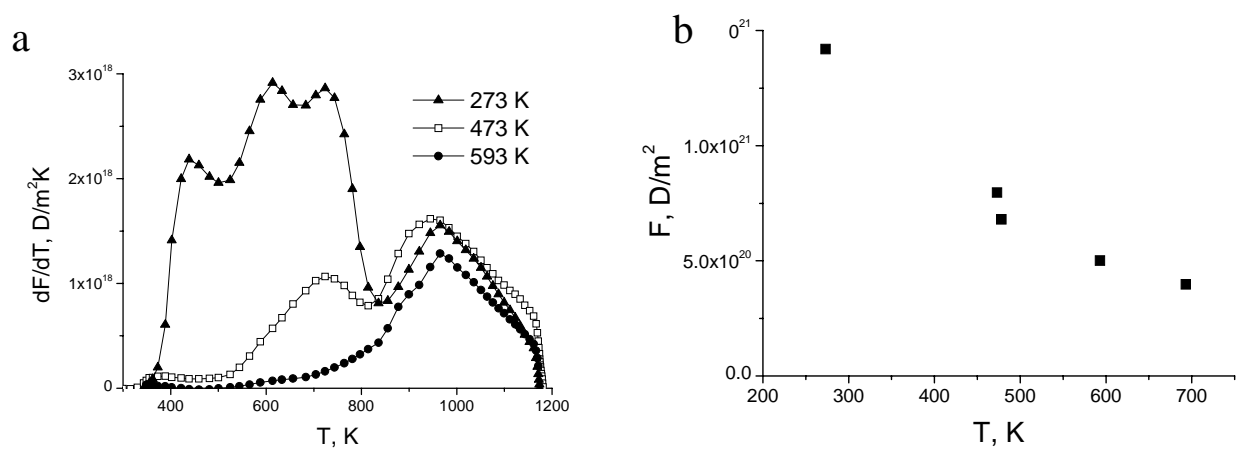

FIGURE 5. Influence of PSW sample temperature during $200 \mathrm{eV} \mathrm{D}{ }^{+}$irradiation on deuterium retention All the fluences were equal $10^{24} \mathrm{D} / \mathrm{m}^{2}$ : a) TDS shapes after deuterium implantation at several sample temperatures; b) temperature dependence of amount deuterium retained.

The first low-energy peak in TDS disappears at $473 \mathrm{~K}$ e.g. deuterium is not trapped in this state any more. Other low-temperature peaks become smaller, while the high temperature peaks remain unchanged.

\section{CONCLUSIONS}

Deuterium retention in plasma-sprayed tungsten in comparison with polycrystalline and monocrystalline tungsten was investigated experimentally by means of thermodesorption spectroscopy (TDS).

An additional high temperature peak at $\mathrm{T} \approx 1150 \mathrm{~K}$ was observed in TDS of PSW in a wide interval of implantation fluencies. The existence of the associated high energy trap site is of significant importance for the use of PSW for fusion reactor applications, because complete removal of tritium from the inner walls of fusion reactors may require temperatures of at least $1250 \mathrm{~K}$. This is almost two times higher than was expected earlier [9].

Deuterium retention in PSW is found to be about 4 times higher than in PCW for incident energies of $200 \mathrm{eV} / \mathrm{D}$ and $3 \mathrm{keV} / \mathrm{D}$ in the fluence interval $10^{22} \div 10^{24} \mathrm{D} / \mathrm{m}^{2}$. The higher retention in PSW has to be taken into account if one thinks of plasma sprayed tungsten for fusion applications. But the deuterium retention in PSW is one order of magnitude lower than in carbon fiber composite (private communication, Dr. J. Roth) which supposed to be used as plasma-facing material in ITER. Thus PSW is still suitable material for ITER.

\section{ACKNOWLEDGMENTS}

One of the authors (A. V. Golubeva) is very grateful to the Max-Planck society for the possibility to perform the experiments. The authors are very grateful to V. Alimov for stimulating the work with plasma-sprayed tungsten. His dictum "this is incredibly interesting!” remains unforgotten and was highly encouraging. The authors want to express their gratitude to A. Weghorn for his technical assistance, and to S. Lindig, G. 
Matern, and J. Perchermaier for their kind help with sample preparation and SEM analysis.

\section{REFERENCES}

1 Federici, G. et al., Nuclear Fusion, Vol. 41 (2001), pp. 1967-2137.

2 Clark, R. E. H., Reiter, D. H., Nuclear Fusion Research, (Springer Verlag, Berin, 2005).

3 Krieger, K., Maier, H. and Neu, R., ASDEX Upgrade Team, Journal of Nuclear Materials Vol. 266269 (1999), pp. 207-216.

4 Mayer, H., Kötterl, S., Krieger, K., Neu, R., Balden, M., ASDEX Upgrade-Team, Journal of Nuclear Materials Vol. 258-263 (1998), pp. 921-926.

5 Neu, R., Rohde, V., Geier, A., Krieger, K., Maier, H., Bolshukhin, D., Kallenbach, A., Pugno, R., Schmidtmann, K., Zarrabian, M., ASDEX Upgrage Team, Journal of Nuclear Materials, Vol. 290293, (2001), pp. 206-210.

6 Deschka, S., García-Rosales, C., Hohenauer, W., Duwe, R., Gauthier, E., Linke, J., Lochter, M., Malléner, W., Plöchl, L., Rödhammer, P. and Salito, A., Journal of Nuclear Materials, Vol. 233237, Part 1 (1996), pp. 645-649.

7 Hildebrandt, D., Akbi, M., Jüttner, B., and Schneider, W., Journal of Nuclear Materials Vol. 266269 (1999), pp. 532-537.

8 Schleußner, D., Maier, H., Franzen, P., Behrisch, R., Balden, M., The ASDEX-Upgrade Team, Perl, M., Knapp, W. and Edelmann, C., Journal of Nuclear Materials Vol. 266-269 (1999), pp. 12961302.

9 García-Rosales, C., Franzen, P., Plank, H., Roth, J. and Gauthier, E., Journal of Nuclear Materials, Vol. 233-237, Part 1 (1996), pp. 803-808.

10 Alimov, V. Kh. and Scherzer, B.M.U., Journal of Nuclear Materials Vol. 240 (1996), pp. 75-80.

11. Eckstein, W., Garcia-Rosales, C., Roth, J., and Ottenberger, W., "Sputtering data”, IPP-report 9/82, 1993.

12 Bay, H.L., Roth, J., and Bohdansky, J., Journal of Applied Physics, Vol. 48, No 11 (1977), pp. $4722-4728$.

13 . Balden, M. and Roth, J., Journal of Nuclear Materials Vol. 280 (2000), p. 39-44.

14 Staudenmaier, G., Roth, J., Behrisch, R., Bohdansky, J., Eckstein, W., Staib, P., Journal of Nuclear Materials Vol. 84, (1979), pp. 149-156.

15 Ogorodnikova, O. V., Mayer, M., Roth, J., Journal of Nuclear Materials Vol. 313-316 (2003), pp. 469-477.

16 Van Veen, A., et al., Journal of Nuclear Materials Vol. 155-157 (1988), pp. 1113-1117.

17 Poon, M., Deuterium trapping in tungsten, thesis, 2004.

18 Alimov, V. Kh., Roth, J., Mayer, M., Journal of Nuclear Materials Vol. 337-339 (2005), pp. 619623. 